\title{
A note on quantum products of Schubert classes in a Grassmannian
}

\author{
Dave Anderson
}

Received: 22 August 2006 / Accepted: 14 September 2006 /

Published online: 5 October 2006

(C) Springer Science + Business Media, LLC 2006

\begin{abstract}
Given two Schubert classes $\sigma_{\lambda}$ and $\sigma_{\mu}$ in the quantum cohomology of a Grassmannian, we construct a partition $\nu$, depending on $\lambda$ and $\mu$, such that $\sigma_{\nu}$ appears with coefficient 1 in the lowest (or highest) degree part of the quantum product $\sigma_{\lambda} \star \sigma_{\mu}$. To do this, we show that for any two partitions $\lambda$ and $\mu$, contained in a $k \times(n-k)$ rectangle and such that the $180^{\circ}$-rotation of one does not overlap the other, there is a third partition $v$, also contained in the rectangle, such that the Littlewood-Richardson number $c_{\lambda \mu}^{v}$ is 1 .
\end{abstract}

Keywords Quantum cohomology · Toric tableau · Littlewood-Richardson number

The purpose of this note is to establish the following fact about the product of classes in the quantum cohomology of a Grassmann manifold:

Proposition 1. If $d$ is the smallest or largest power of $q$ appearing in the quantum product $\sigma_{\lambda} \star \sigma_{\mu}$, then there exists a Schubert class $\sigma_{v}$ such that the Gromov-Witten invariant $c_{\lambda \mu}^{v}(d)$ is equal to 1 .

In fact, we will explicitly construct such a class. The main idea is to use a result of Postnikov (Corollary 8.4 in [5]), which equates these Gromov-Witten invariants to certain classical Littlewood-Richardson numbers. The above proposition then follows from a statement about classical cohomology (Proposition 3 below), which says that whenever $\lambda$ and $\mu$ are such that $\sigma_{\lambda} \cdot \sigma_{\mu} \neq 0$, one can construct a partition $v$ such that $c_{\lambda \mu}^{\nu}=1$. Moreover, we conjecture that the result holds for all powers of $q$ appearing in $\sigma_{\lambda} \star \sigma_{\mu}$. We conclude with a comment on an application of this fact to "real quantum cohomology."

D. Anderson $(\bowtie)$

Department of Mathematics, University of Michigan, Ann Arbor, MI 48109

e-mail: dandersn@umich.edu 
Before discussing Postnikov's result and the construction of the class $\sigma_{\nu}$, we recall some basic definitions, notation, and results related to quantum cohomology of Grassmannians. Let $X=G r_{k}\left(\mathbb{C}^{n}\right)$ be the Grassmannian of $k$-planes in $\mathbb{C}^{n}$. The cohomology ring $H^{*}(X ; \mathbb{Z})$ is well-understood. It has a linear basis of Schubert classes $\sigma_{\lambda}$, indexed by partitions whose Young diagrams fit inside the $k$-by- $(n-k)$ rectangle; these classes correspond to the Schubert varieties $\Omega_{\lambda}$ of codimension $|\lambda|=\#($ boxes in $\lambda$ ). (Thus the class $\sigma_{\lambda}$ has degree $2|\lambda|$.) The structure constants for multiplication in this basis are the Littlewood-Richardson numbers $c_{\lambda \mu}^{\nu}$-that is,

$$
\sigma_{\lambda} \cdot \sigma_{\mu}=\sum_{\nu} c_{\lambda \mu}^{\nu} \sigma_{\nu}
$$

The (small) quantum cohomology ring $Q H^{*}(X)$ is a module over the polynomial ring $\mathbb{Z}[q]$, where $q$ is a formal variable of degree $n$, with a corresponding $\mathbb{Z}[q]$-basis of Schubert classes $\sigma_{\lambda}$. The ring structure is given by quantum multiplication, denoted by ' $\star$ ', which has for structure constants the (three-point, genus 0) Gromov-Witten invariants. That is,

$$
\sigma_{\lambda} \star \sigma_{\mu}=\sum_{d} q^{d} \sum_{\nu} c_{\lambda \mu}^{\nu}(d) \sigma_{\nu}
$$

where $c_{\lambda \mu}^{\nu}(d)$ is, by definition, the number of degree- $d$ rational curves passing through general translates of $\Omega_{\lambda}, \Omega_{\mu}$, and $\Omega_{v^{\vee}}$; by degree considerations, it is nonzero only when $|\lambda|+|\mu|=|\nu|+d n$.

The ring $Q H^{*}(X)$ has been much-studied in recent years; we mention only a few results most relevant to this note. Agnihotri showed that the quantum product $\sigma_{\lambda} \star \sigma_{\mu}$ is never zero (see [1], Section 5); Fulton and Woodward gave a characterization of the lowest power of $q$ appearing in $\sigma_{\lambda} \star \sigma_{\mu}$, and generalized this to all $G / P$ [3]; Yong gave an upper bound for the powers of $q$ appearing in a quantum product and conjectured that these powers form an unbroken sequence from lowest to highest; Postnikov refined the results of [3] for type $A$, gave a formula for equating the Gromov-Witten invariants $c_{\lambda \mu}^{v}(d)$ to Littlewood-Richardson numbers when $d$ is the minimal or maximal power of $q$ appearing in $\sigma_{\lambda} \star \sigma_{\mu}$, and proved Yong's conjecture [5].

Now we introduce some notation, following [5]. All partitions will lie inside the $k$-by- $(n-k)$ rectangle. If we draw the diagram of a partition $\lambda$ inside the rectangle, the border traces a path from the $S W$ corner to the $N E$ corner of the rectangle; the 01 -word $\omega(\lambda)$ is the $n$-digit string which assigns a " 0 " to each step right, and a " 1 " to each step up. Writing $\omega(\lambda)=\left(\omega_{1}, \ldots, \omega_{n}\right)$, define a doubly infinite integer sequence $\phi=\phi(\lambda)=\left(\phi_{i}\right)_{i \in \mathbb{Z}}$ by $\phi_{i}=\omega_{1}+\cdots+\omega_{i}$ for $1 \leq i \leq n$, and $\phi_{i+n}=\phi_{i}+k$ for all $i$. Also, let $\lambda^{\vee}$ denote the complement of $\lambda-$ that is, $\lambda^{\vee}=\left(n-k-\lambda_{k}, \ldots, n-k-\lambda_{1}\right)$ - and let $\lambda^{\prime}$ be the conjugate of $\lambda$. Here is an example, for $k=5$ and $n=11$ :

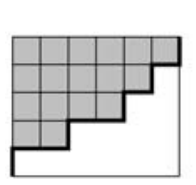

$$
\begin{aligned}
& \lambda=(6,5,4,2) \\
& \lambda^{\vee}=(6,4,2,1) \\
& \lambda^{\prime}=(4,4,3,3,2,1) \\
& \omega(\lambda)=(1,0,0,1,0,0,1,0,1,0,1) \\
& \phi(\lambda)=(\ldots, 1,1,1,2,2,2,3,3,4,4,5, \ldots)
\end{aligned}
$$


Define the cyclic rotation $S^{i}(\lambda)$ to be the partition whose 01 -word is obtained from $\omega(\lambda)$ by cyclically permuting $i$ places to the left (or $-i$ places to the right, if $i$ is negative). For instance, with $\lambda=(6,5,4,2)$, we have $\omega(\lambda)=(1,0,0,1,0,0,1,0,1,0,1)$, so $\omega\left(S^{2}(\lambda)\right)=(0,1,0,0,1,0,1,0,1,1,0)$, and thus $S^{2}(\lambda)=(5,5,4,3,1)$. Finally, given two partitions $\lambda$ and $\mu$, define integers $D_{\min }$ and $D_{\max }$ by

$$
\begin{aligned}
D_{\min } & =-\min _{i}\left\{\phi_{i}(\lambda)+\phi_{-i}(\mu)\right\} \\
D_{\max } & =-\max _{i}\left\{\phi_{-i}(\lambda)+\phi_{i-(n-k)}(\mu)\right\} .
\end{aligned}
$$

Of course, it suffices to consider $1 \leq i \leq n$ in this definition, since the sequences $\left\{\phi_{i}(\lambda)+\phi_{-i}(\mu)\right\}$ and $\left\{\phi_{-i}(\lambda)+\phi_{i-(n-k)}(\mu)\right\}$ are $n$-periodic.

The meaning of these definitions becomes clearer in the language of Postnikov's toric shapes. (We will not need these notions for the proof of Proposition 1, but we will use them to formulate our conjecture for intermediate powers of $q$.) Consider the lattice $\mathbb{Z}^{2}$ in the plane, with matrix coordinates; i.e., the point $(i, j)$ is $i$ steps down and $j$ steps right from the origin. Let $R_{k n}$ be the rectangle with vertices $(0,0),(k, 0)$, $(0, n-k)$, and $(k, n-k)$, and let the cylinder $C_{k n}$ be the quotient $\mathbb{Z}^{2} / \mathbb{Z} \cdot(-k, n-k)$. (Thus the SW and NE corners of $R_{k n}$ are identified in $C_{k n}$.) If $\lambda$ is a partition inside $R_{k n}$, the cylindric loop $\lambda[0]$ is the image of the border of $\lambda$ in $C_{k n}$. The shifted cylindric loop $\lambda[d]$ is the translation of $\lambda[0]$ by $(d, d)$. We will often identify $\lambda[d]$ with its preimage in the plane; this is just the periodic continuation of the (translated) border of $\lambda$. See Fig. 1.

A frame is any translation of $R_{k n}$ in the plane, and the anchor of a frame is its SW corner. If we move a frame so that its anchor lies on $\lambda[0]$, then the part of $\lambda[0]$ contained inside the frame forms the border of a partition. In fact, if the anchor is shifted $i$ steps in the NE direction along $\lambda[0]$, then the resulting partition is $S^{i}(\lambda)$. Also, the number $\phi_{i}(\lambda)$ is the vertical distance traveled after $i$ steps NE along $\lambda[0]$ (so the frame for $S^{i}(\lambda)$ is translated up by $\phi_{i}(\lambda)$ from $R_{k n}$, and right by $i-\phi_{i}(\lambda)$ ). See Fig. 2.

If $\lambda$ and $\mu$ are partitions such that $\mu[d]$ is (weakly) right and below $\lambda[0]$ in the plane, so that the region between $\mu[d]$ and $\lambda[0]$ forms a connected strip, then the image of this region in $C_{k n}$ is called a cylindric shape and denoted $\mu / d / \lambda$. Let $\lambda^{\downarrow}\left[0^{\downarrow}\right]$ denote the translation of $\lambda[0]$ by $(k, 0)$. A cylindric shape $\mu / d / \lambda$ is toric if $\mu[d]$ lies between

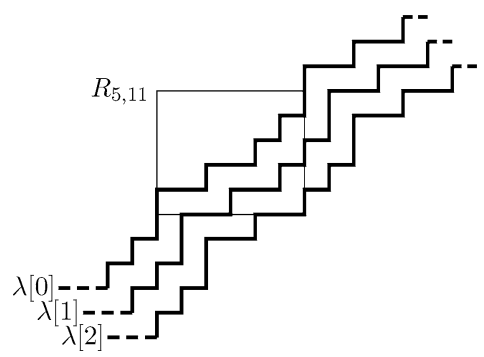

Fig. 1 Cylindric loops, for $\lambda=(6,5,4,2)$ 


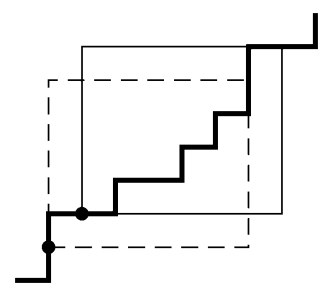

Fig. 2 Rotating the frame

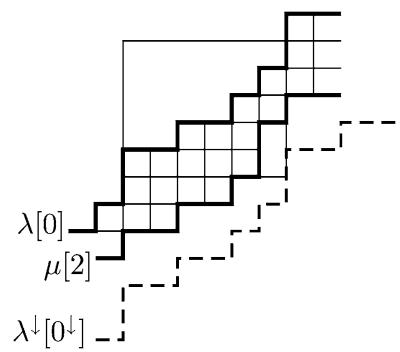

Fig. 3 The toric shape $\mu / 2 / \lambda$, for $\mu=(4,3,3,2)$ and $\lambda=(6,5,4,2)$

$\lambda[0]$ and $\lambda^{\downarrow}\left[0^{\downarrow}\right]$ (Fig. 3). It is not hard to see that the numbers $D_{\min }$ and $D_{\max }$ defined above are the minimum and maximum values of $d$ such that $\mu^{\vee} / d / \lambda$ is a toric shape.

Postnikov shows that $q^{d}$ appears in the quantum product $\sigma_{\lambda} \star \sigma_{\mu}$ if and only if $\mu^{\vee} / d / \lambda$ is a toric shape, and deduces that $D_{\min }$ and $D_{\max }$ are, respectively, the minimum and maximum powers of $q$ appearing in this product. By the definitions, there are integers $a$ and $b$ such that $D_{\min }+\phi_{a}(\lambda)+\phi_{-a}(\mu)=0$ and $D_{\max }+\phi_{-b}(\lambda)+$ $\phi_{b-(n-k)}(\mu)=0$. (There may be more than one such $a$ and $b$, but any choice will do.) Set

$$
\begin{aligned}
& \lambda^{\min }=S^{a}(\lambda), \\
& \mu^{\min }=S^{-a}(\mu), \\
& \lambda^{\max }=S^{b}\left(\lambda^{\vee}\right), \quad \text { and } \\
& \mu^{\max }=S^{n-k-b}\left(\mu^{\vee}\right) .
\end{aligned}
$$

Then Postnikov proves the following:

Proposition 2 ([5], Corollary 8.4). Let $v$ be any partition in the $k-b y-(n-k)$ rectangle. Then

$$
\begin{aligned}
& c_{\lambda \mu}^{v}\left(D_{\min }\right)=c_{\lambda^{\min } \mu^{\min }}^{v}, \quad \text { and } \\
& c_{\lambda \mu}^{\nu}\left(D_{\max }\right)=c_{\lambda^{\max } \mu^{\max } .}^{\nu^{\vee}}
\end{aligned}
$$


In particular, the products $\sigma_{\lambda^{\min }} \cdot \sigma_{\mu^{\min }}$ and $\sigma_{\lambda_{\max }} \cdot \sigma_{\mu^{\max }}$ are nonzero in $H^{*}(X)$.

By substituting $\lambda$ for $\lambda^{\mathrm{min}}$, and so on, this reduces Propostion 1 to the following:

Proposition 3. Let $\lambda$ and $\mu$ be any partitions contained in the $k$-by-( $n-k)$ rectangle, such that $\sigma_{\lambda} \cdot \sigma_{\mu} \neq 0$ in $H^{*}(X)$. Then there is a partition $v=v(\lambda, \mu)$, also contained in the rectangle, such that $c_{\lambda \mu}^{\nu}=1$.

If we write $\mu_{180}$ for the $180^{\circ}$-rotation of $\mu$ inside the $k$-by- $(n-k)$ rectangle, note that the condition $\sigma_{\lambda} \cdot \sigma_{\mu} \neq 0$ is equivalent to requiring that $\lambda$ and $\mu_{180}$ do not overlap. (This notation should cause no confusion, as we will not discuss partitions with 180 parts.) Note that the boxes of $\mu_{180}$ form the complement of $\mu^{\vee}$ inside the rectangle.

Proof: We will construct the partition $v$, and use the following version of the Littlewood-Richardson rule: The number $c_{\lambda \mu}^{\nu}$ is equal to the number of semistandard Young tableaux of shape $\nu / \lambda$ with reading word of type $\mu .{ }^{1}$ We will call such a tableau on $\nu / \lambda$ a Littlewood-Richardson filling of type $\mu$. (See [2] or [6, Appendix 1] for more on the Littlewood-Richardson rule.)

Draw $\lambda$ and $\mu_{180}$ inside the rectangle. Now slide the columns of $\mu_{180}$ up against $\lambda$, and then left-justify all rows. The resulting shape is $v(\lambda, \mu)$. Here is an example, with $k=5, n=11, \lambda=(4,3,1)$, and $\mu=(5,4,4)$. (The shape of $\lambda$ is shaded, and that of $\mu_{180}$ is filled by numbers.)

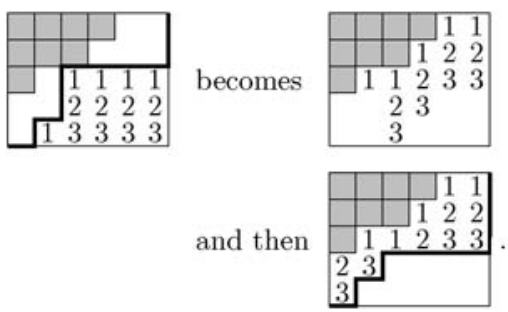

In this example, then, $v(\lambda, \mu)=(6,6,6,2,1)$.

This sliding algorithm is reminiscent of the moves in Schützenberger's jeu de taquin [7] (see also [6, Appendix 1]). In fact, the bulk of the sliding described here can be accomplished via jeu-de-taquin moves; however, as the above example shows, it is not exactly the same as jeu de taquin. (In jeu de taquin, the ' 3 ' in the bottom row would slide up, and the final shape would be $(6,6,6,3)$.)

Numerically, let $\rho$ be the partition formed by sorting

$$
\left(k-\lambda_{1}^{\prime}-\mu_{n-k}^{\prime}, k-\lambda_{2}^{\prime}-\mu_{n-k-1}^{\prime}, \ldots, k-\lambda_{n-k}^{\prime}-\mu_{1}^{\prime}\right)
$$

\footnotetext{
${ }^{1}$ The reading word of a tableau is the integer string formed by reading the entries of the tableau from right to left, starting at the top row. A word $w=w_{1} w_{2} \cdots w_{p}$ is of type $\mu$ if one can build the diagram of $\mu$ by placing a box in row $w_{1}$, then in row $w_{2}$, etc., in such a way that one has a Young diagram at each step. The condition that each stage be a Young diagram is equivalent to requiring that for each $m \leq p$,
}

$$
\#\left(1 \text { 's in }\left\{w_{1}, \ldots, w_{m}\right\}\right) \geq \#\left(2 \text { 's in }\left\{w_{1}, \ldots, w_{m}\right\}\right) \geq \cdots ;
$$

this is called the Yamanouchi condition. 
(These are the heights of the spaces between the columns of $\lambda$ and $\mu_{180}$.) The sliding construction described above leaves the shape $\left(\rho^{\prime}\right)_{180}$ in the bottom right corner. Indeed, sliding $\mu_{180}$ up leaves blank columns of heights $\left(k-\lambda_{i}^{\prime}-\mu_{n-k+1-i}^{\prime}\right)$, and left-justifying the filled space is the same as right-justifying blank space, which is equivalent to sorting. Thus $v(\lambda, \mu)=\left(\rho^{\prime}\right)^{\vee}$. In the above example, $\rho=(2,2,2,2,1)$, so $\rho^{\prime}=v^{\vee}=(5,4)$.

Now we must show that $c_{\lambda \mu}^{\nu}=1$. First, we exhibit a Littlewood-Richardson filling of $v / \lambda$, proving $c_{\lambda \mu}^{v} \geq 1$. In fact, the tableau produced in our running example is a Littlewood-Richardson filling; we claim the procedure suggested there works in general. Let us make this precise. Consider $\mu_{180}$ as a skew shape, and fill its boxes by writing the numbers $1,2,3, \ldots$ down columns, so that the $r$ th column from the right has entries $1,2, \ldots, \mu_{r}^{\prime}$. Note that this is a Littlewood-Richardson filling of type $\mu$. Now slide the boxes as prescribed (first moving them up against $\lambda$, then left-justifying), carrying their labels along. The result is, by definition, a tableau on the shape $v / \lambda$.

We need to check that the result is actually a Littlewood-Richardson filling of type $\mu$. By construction, the tableau has entries corresponding to $\mu$. The sliding operations preserve weak increase along rows and strict increase down columns, so the tableau is semistandard. It remains to verify the Yamanouchi condition; for this, we will consider the intermediate shape $\theta$ formed by sliding $\mu_{180}$ up against $\lambda$, and the corresponding filling of $\theta$-this is obtained by filling the columns of $\theta$ just as was done with $\mu_{180}$, so that the $r$ th column from the right has entries $1, \ldots, \mu_{r}^{\prime}$. Note that the reading word is unchanged by left-justification, so it suffices to show that the reading word of this filling ( of $\theta$ ) satisfies the Yamanouchi condition.

Let $B$ be the $m$ th box one reads when forming the reading word $w$. The letters $w_{1}, \ldots, w_{m}$ are the entries appearing in rows strictly above $B$, or in the same row and weakly right of $B$. In Fig. 4, $B$ is the darkly shaded box, and the entries in question are all those in the shaded region. Every entry in a given column is distinct, so the number of $i$ 's apearing in the shaded region is bounded by the number of columns in the shaded region. There is a 1 at the top of each column, so we see that

$$
\#(1 \text { 's })=\#(\text { columns }) \geq \#(i \text { 's })
$$

for each $i>1$. If we remove the boxes filled with 1's, we can repeat this argument on the part of the shaded region that remains; this shows that the Yamanouchi condition holds.

One can prove the reverse inequality $c_{\lambda \mu}^{\nu} \leq 1$ by pondering tableaux, but here is a simpler way, pointed out to me by Sergey Fomin. Let $\rho$ be the sorting of the numbers $\left(k-\lambda_{i}^{\prime}-\mu_{n-k+1-i}^{\prime}\right)$, as above. First, note that $\rho_{1}^{\prime}$ is the size of the (unique) largest

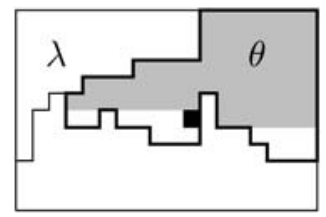

Fig. 4 Verifying the Yamanouchi condition 


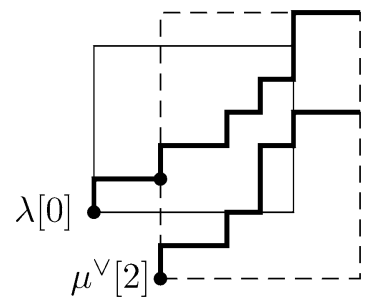

Fig. 5 Setup for sliding, with $\lambda=(6,5,4,2)$ and $\mu=(6,4,3,3,2)$

horizontal strip which can be added to $\lambda$ without overlapping $\mu_{180}$ or spilling outside the rectangle. Indeed, $\rho_{1}^{\prime}$ is the number of nonzero parts of $\rho$, which is the number of columns (of the rectangle) in which there is space between $\lambda$ and $\mu_{180}$. It follows (by Pieri's rule) that $\sigma_{\lambda} \cdot \sigma_{\rho_{1}^{\prime}} \cdot \sigma_{\mu}=\sigma_{\tilde{\lambda}} \cdot \sigma_{\mu}$, where $\tilde{\lambda}$ is the shape formed by adding this longest horizontal strip to $\lambda$. If we write $\tilde{\rho}$ for the partition formed from the vertical spaces between $\tilde{\lambda}$ and $\mu_{180}$, then $\tilde{\rho}_{1}^{\prime}=\rho_{2}^{\prime}$. Proceeding inductively, we see that

$$
\sigma_{\lambda} \cdot\left(\sigma_{\rho_{1}^{\prime}} \cdots \cdot \sigma_{\rho_{s}^{\prime}}\right) \cdot \sigma_{\mu}=\sigma_{\mu^{\vee}} \cdot \sigma_{\mu}=1 \cdot[p t] .
$$

It follows that $c_{\lambda \mu}^{\alpha^{\vee}} \leq 1$ for every partition $\alpha$ appearing in the Schubert expansion of $\left(\sigma_{\rho_{1}^{\prime}} \cdots \sigma_{\rho_{s}^{\prime}}\right)$. Since $\rho^{\prime}$ is such a partition, and $v=\left(\rho^{\prime}\right)^{\vee}$, we are done.

We conclude by describing a conjectured algorithm for producing a class $v=$ $\nu(\lambda, \mu, d)$, for each $d$ between $D_{\min }$ and $D_{\max }$, such that $c_{\lambda \mu}^{\nu}(d)=1$. Begin by drawing the paths $\lambda[0]$ and $\mu^{\vee}[d]$; mark the point on $\mu^{\vee}[d]$ which is the translation of the anchor by $(d, d)$. (See Fig. 5.) Consider the box formed by the union of two frames: one whose anchor is at $(d, d)$, and the other whose anchor is at the point of $\lambda[0]$ directly above $(d, d)$. Perform the sliding algorithm described in the proof of Proposition 3 for the shapes whose borders are the parts of $\lambda[0]$ and $\mu^{\vee}[d]$ lying inside this box. Call the partition produced by the sliding algorithm $\tilde{v}$, and let $v(\lambda, \mu, d)$ be the partition formed by the last $k$ parts of $\tilde{v}$ (including zeroes). (This is the part of $\tilde{v}$ lying inside the frame whose anchor is at $(d, d)$.)

For example, with $k=5, n=11, \lambda=(6,5,4,2)$, and $\mu=(6,4,3,3,2)$, the algorithm produces $v(\lambda, \mu, 2)=(6,6,1)$ :

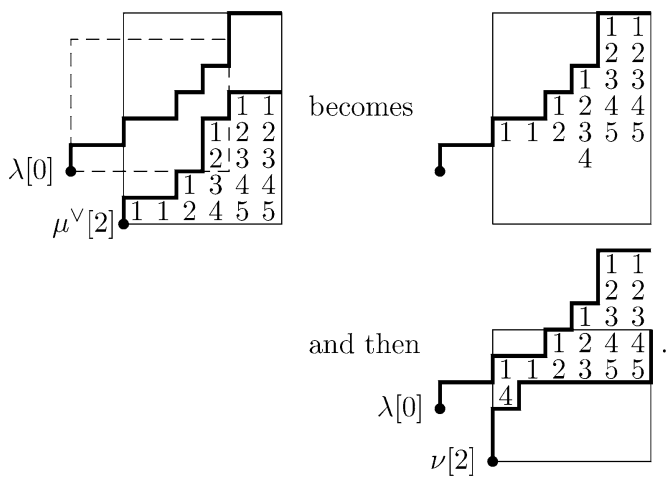


One can check that $c_{(6,5,4,2),(6,4,3,3,2)}^{(6,6)}(2)=1$. We conjecture that this always works: if $v=v(\lambda, \mu, d)$ is as described above, for $D_{\min } \leq d \leq D_{\max }$, then $c_{\lambda \mu}^{v}(d)=1$. In particular, we expect the following generalization of Proposition 1 to hold:

Conjecture 4. If $d$ is any power of $q$ appearing in the quantum product $\sigma_{\lambda} \star \sigma_{\mu}$, then there exists a Schubert class $\sigma_{\nu}$ such that the Gromov-Witten invariant $c_{\lambda \mu}^{\nu}(d)$ is equal to 1 .

To summarize, we have seen that

$$
c_{\lambda \mu}^{\nu\left(\lambda^{\min }, \mu^{\min }\right)}\left(D_{\min }\right)=c_{\lambda^{\min }, \mu^{\min }}^{\nu\left(\mu^{\min }\right)}=1
$$

and

$$
c_{\lambda \mu}^{\nu\left(\lambda^{\max }, \mu^{\max }\right)^{\vee}}\left(D_{\max }\right)=c_{\lambda^{\max }, \mu^{\max }}^{\nu\left(\lambda^{\max } \mu^{\max }\right)}=1 .
$$

Of course, this implies that the mod 2 reduction of $\sigma_{\lambda} \star \sigma_{\mu}$ is always nonzero. This can be seen as an analogue of one of the main results of [3] for "mod 2 real quantum Schubert calculus," at least for Grassmannians. ${ }^{2}$ Similarly, a proof of Conjecture 4 would establish a real analogue of the stronger result that the powers of $q$ appearing in a quantum product form an unbroken sequence from $D_{\min }$ to $D_{\max }$ [5, Theorem 8.1].

I would like to thank William Fulton for suggesting this question and for comments on the manuscript, and Sergey Fomin for a helpful discussion. Anders Buch's Littlewood-Richardson calculator ${ }^{3}$ proved invaluable for experimentation.

\section{References}

1. A. Bertram, I. Ciocan-Fontanine, and W. Fulton, "Quantum multiplication of Schur polynomials," J. Algebra 219 (1999), 728-746.

2. W. Fulton, Young Tableaux, Cambridge Univ. Press, 1997.

3. W. Fulton and C. Woodward, "On the quantum product of Schubert classes," J. Algebraic Geom. 13 (2004), 641-661.

4. S. Kwon, "Real aspects of the moduli space of genus zero stable maps and real version of the Gromov-Witten theory," math.AG/0305128.

5. A. Postnikov, "Affine approach to quantum Schubert calculus," Duke Math. J. 128(3) (2005), 473-509.

6. R.P. Stanley, Enumerative Combinatorics, Volume 2, with appendix by S. Fomin, Cambridge, 1999.

7. M.P. Schützenberger, "La correspondance de Robinson," in Combinatoire et Represéntation du Groupe Symétrique, Lecture Notes in Math., 579 (1977), Springer-Verlag, 59-135.

8. A. Yong, "Degree bounds in quantum Schubert calculus," Proc. Amer. Math. Soc. 131(9) (2003), 2649-2655.

\footnotetext{
${ }^{2}$ The phrase in quotes should be interpreted as follows: Let $\bar{M}=\bar{M}_{0,3}(X, d)$ be the Kontsevich moduli space of stable maps, and let $\bar{M}(\mathbb{R})$ be its real part. The Gromov-Witten invariants $c_{\lambda \mu}^{v}(d)$ are certain intersection numbers in $H^{*}(\bar{M}, \mathbb{Z})$; let $\bar{c}_{\lambda \mu}^{v}(d)$ be the analogous intersection numbers in $H^{*}(\bar{M}(\mathbb{R}), \mathbb{Z} / 2 \mathbb{Z})$. It is reasonable to expect that $\bar{c}_{\lambda \mu}^{v}(d) \equiv c_{\lambda \mu}^{v}(d)(\bmod 2)$, as is true for the classical case $(d=0)$. An outline discussion of intersection theory on $\bar{M}(\mathbb{R})$ can be found in [4].

${ }^{3}$ Available at http: //www.math.rutgers . edu/ asbuch/lrcalc/. 